\title{
Zweitmeinung aus dem Netz - das rechtliche Eis ist noch dünn
}

\author{
Sollen Patienten vor einer Operation regelmäßig eine Zweitmeinung \\ einholen? Kann das ohne direkten Kontakt zum Arzt etwas bringen? \\ Wo beginnt das Fernbehandlungsverbot? Für Ärzte, die bereit wären, \\ in Zweifelsfällen eine Zweitmeinung anzubieten, stellen sich viele \\ rechtliche Fragen. Und ein genauer Blick zeigt: Das Recht hinkt der \\ Zeit hinterher.
}

$\mathrm{N}$ eue Online-Angebote wie das Portal vorsicht-operation.de geben Patienten die Möglichkeit, eine Zweitmeinung einzuholen, etwa im Vorfeld einer Operation. Hier stehen Experten zur Verfügung, die Einzelfälle begutachten. Der Patient kann über das Portal einen Fragebogen ausfüllen und Befunde elektronisch einsenden. Auf Grundlage dieser Angaben wird ein Gutachten mit Diagnose und Therapieempfehlungen sowie alternativen Behandlungsmöglichkeiten erstellt.

\section{MBO-Ä im Umbruch}

Die Angebote traditioneller Portale bestehen bisher etwa in Konsilien unter Kollegen, in der Erteilung therapeutischer Ratschläge an den Patienten durch seinen behandelnden Arzt, in Notfällen oder im Rahmen der Erbringung telemedizinischer Leistungen. Konkrete Diagnosen oder

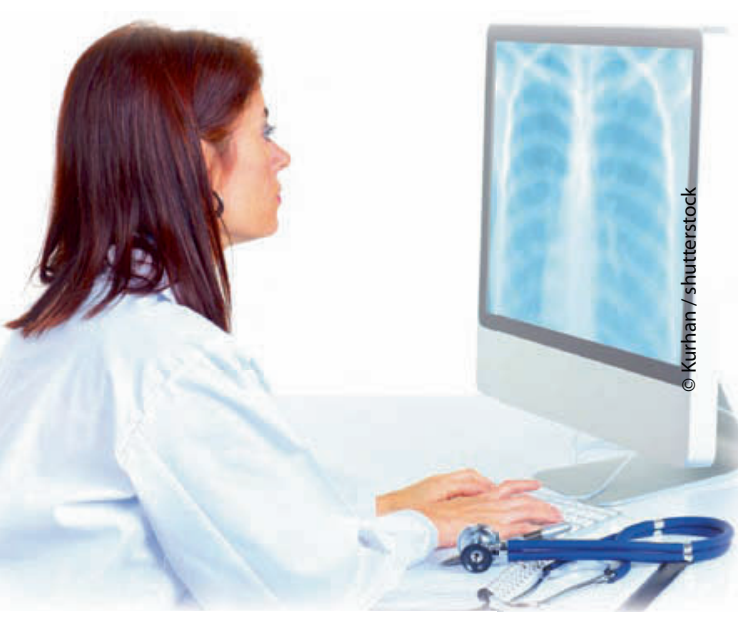

Therapieempfehlungen werden dabei meist nicht abgegeben, die Behandlungsverantwortung bleibt beim behandelnden (überweisenden) Arzt. Die Anforderungen an eine sichere Kommunikation sind bekannt und können eingehalten werden.

Neue Fragen stellen sich aber in berufsund haftungsrechtlicher Hinsicht. Derzeit dürfen Ärzte individuelle ärztliche Behandlung, insbesondere auch Beratung, „weder ausschließlich brieflich noch in Zeitungen oder Zeitschriften noch ausschließlich über Kommunikationsmedien oder Computerkommunikationsnetze durchführen" ( $\$ 7$ Abs. 3 MBO-Ä). Die Vorschrift will den Einsatz der genannten Medien nicht gänzlich unterbinden, über die Reichweite des Verbots besteht aber auch in der geplanten Änderungsfassung kaum Klarheit. Darf die „Fernbehandlung" nur vom Erstbehandler, der den Patienten persönlich gesehen hat, durchgeführt werden? Ist die Grenze bei Diagnosestellung oder Therapieempfehlung nur auf Grundlage von Röntgen-/MRTBildern und Fragebögen zu ziehen? Bedarf es der Zusammenarbeit zwischen Erstbehandler und Zweitmeinungsgeber? Womöglich werden die Antworten der Berufsgerichte zunächst restriktiv sein, später aber vom Bundesverfassungsgericht liberalisierend korrigiert werden.

Haftungsrechtlich kann sich ein Konsiliararzt auf die Aussagen seines überweisenden Kollegen im Regelfall verlassen

Befundung via Internet: „Die Zeit ist reif, ist es das Recht auch?" und darauf, dass dieser den Patienten ordnungsgemäß untersucht hat. Zweitmeinungsportale werden aber unmittelbar vom Patienten in Anspruch genommen und das im Zweifel ohne Zustimmung oder Kenntnis des Erstbehandlers. Ihm werden Diagnosen gestellt und konkrete Behandlungsmöglichkeiten aufgezeigt. Erhält ein Arzt eine Anfrage ausschließlich elektronisch, gelten andere Bewertungsmaßstäbe. Der die Diagnose stellende oder eine Therapieempfehlung abgebende Arzt kann sich nicht auf das Privileg eines Konsiliararztes berufen. Er haftet selbst und umfänglich für mögliche Fehlleistungen aus einem Behandlungsvertrag mit dem Patienten. Den Arzt wird hier außerdem die Pflicht treffen, den Patienten vollständig therapeutisch aufzuklären. Soweit ist die Rechtslage klar.

\section{Neue medizinische Dienstleistung - die Online-Welt ist bereit}

Zweitmeinungen werden von der Politik immer wieder gefordert, sie sind wichtig und nicht neu. Sie auf einem Portal anzubieten passt in unsere Online-Welt. Das geltende Recht kann sich dieser neuen medizinischen Dienstleistung öffnen, wenn es gleichzeitig das Wohl des Patienten sicherstellt. Warum soll der mündige Patient nicht eine Online-Zweitmeinung in Anspruch nehmen, wenn er die womöglich bestehenden Defizite einer nicht-persönlichen Beratung akzeptiert? Die Rechtsprechung des BGH akzeptiert schließlich auch den Aufklärungsverzicht, wenn das Wesentliche der Aufklärung kommuniziert wird und der Patient weiß, dass er auf Teilinhalte verzichtet. Bei entsprechender Aufklärung und Einwilligung im Online-Portal kann die Rechtsgrundlage belastbar gestaltet werden. Mit anderen Worten: Die Zeit ist reif für ein interaktives Zweitmeinungs-Portal, aber ist das Recht es auch?

Christian Dierks, Facharzt für Allgemeinmedizin und Fachanwalt für Medizinrecht und Sozialrecht in Berlin 